\title{
Characterization of industrial catalysts by EXAFS
}

J. Lynch

Institut Français du Pétrole, 1 et 4 Ave. de Bois Preau, 92500 Rueil Malmaison, France

\begin{abstract}
Industrial catalysts are designed to present very high specific surface areas and are thus in general poorly crystallised, being often amorphous to X-ray diffraction for instance. In addition the working conditions of these solids are severe, high temperatures and pressures of reactive gases are used during both use and preparation. The penetrating power of X-rays enables in-situ devices to be built so that the the processes involved in activation and deactivation of catalysts can be followed. Several families of industrial catalysts are easily amenable to $\mathrm{X}$-ray absorption spectroscopy analysis. These include sulfide based catalysts, typically with over ten weight percent metal content. Problems are encountered in the study of precious metal systems, for example platinum based bimetallic catalysts supported on aluminium oxide,where metal loading is lower ( less than one weight percent ). Using in-situ conditions enables studies of the dynamics of reactions and of the state of working catalysts. The structural modifications observed indicate that this type of study is often necessary to obtain meaningful relationships between the state of the solid and its chemical behaviour.
\end{abstract}

\section{INTRODUCTION}

Catalysis is a vast field of research. The present paper will only cover a small part of the field, concerned with industrial heterogeneous catalysts. For industrial processes the catalyst is deliberately " designed ", usually from knowledge of the functionnement of already existing catalysts, to meet a specific need and is built from well controlled starting materials. Heterogeneous catalysis concerns applications in which the catalyst is in a different state ( usually solid) to the reactants and products ( which may be gases or liquids ). This excludes for example natural biological catalysis but also homogeneous industrial catalysis using organometallic molecules. Aside from the obvious structural differences of heterogeneous catalysts, there are general differences in the conditions under which the latter are used and in their performances which make them more difficult to investigate. Heterogeneous catalysis typically takes place under conditions of high temperature and pressure. Often several functions such as selective bond breaking and cycle formation need to be performed by the same solid which will then be multi - component. Thus, although the starting materials are well caracterised, the end product is hard to control.

Despite these difficulties, investigation of the environnement of active species in catalysts was one of the first applications of X-ray Absorption spectroscopy ( XAS )[1]. The potential of the technique was realised so early on partly because few classical methods allow access to structural and electronic information about these systems. The incentive to obtain this information comes from the industrial need to better understand how these systems work and to improve their performances. World consumption of catalysts has reached $\$ 10^{10}$ per year, about half of which is dedicated to environnemental clean - up such as automobile exhaust control. The size of the market is partly due to the frequent use of precious metals, platinum is a classic example, in the formulations. On this scale, even a one per cent increase in efficiency represents a significant sum of money. The 400,000 tons of catalyst produced each year do not have an unlimited life : although they can be and are regenerated several times, on replacement they 
constitute a potential polluant. They have to be treated to recover the metals and the poisons such as sulfur accumulated during use. The possibility of replacing existing catalytic solids by cheaper and less polluting materials is very attractive. In order to achieve this however, a better understanding of how the catalyst works is essential.

\section{THE STRUCTURE OF INDUSTRIAL CATALYSTS}

Industrial catalysts cover an extremely wide range of compositions and structures, optimised as a function of the reaction to be promoted. Two major classes which will be illustrated here are :

- Supported sulfides for desulfuration of heavy crude oils. The archetype consists of $\mathrm{MoS}_{2}$ supported on alumina. Of major importance in this field are the addition of promotors such as Ni or Co which have been found to improve the hydrogenation rate of the desulfurised molecules.

- Supported metals, used for instance in reforming. Again there is a classic example, small particles of platinum supported by alumina. In the last few decades bimetallic systems have been industrialised such as $\mathrm{Pt}-\mathrm{Re}, \mathrm{Pt}-\mathrm{Sn}$ etc. which have improved performances such as resistance to poisoning by sulfur in the charge (for Pt-Re) or stability of the particle sizes during regeneration (Pt-Sn). One of the common factors of these systems is their high specific surface. Catalysis, involving adsorption of the reactants and desorption of the products, is a surface reaction so that bulk atoms do not in general participate. The aluminium oxides used as support material are the transition ( hydrated ) phases such as gamma alumina which cristallises as small plates a few tens of nanometres in size with a corresponding specific surface of several hundred square metres per gramme.

During the activation and use of these catalysts they come into contact with a number of gas mixtures. The impregnated oxide phases are first dried and calcined in air at high temperature to ensure stability. Activation occurs under hydrogen for metallic catalysts, under hydrogen disulfide for the formation of a sulfide phase. Under reaction conditions, in contact with hydrocarbons, the catalyst may suffer deactivation from a deposit of condensed polyaromatic carbon or coke. The catalyst can be regenerated only by burning off the carbon in air at high temperatures, resulting once again in the formation of an oxyde phase. An industrial catalyst may go through several cycles of oxydation and reduction before it is replaced. This leads to a second type of deactivation due to sintering of the particles, resulting in loss of surface area.

Metal particles need to be small, in general subnanometre in diametre, in order to avoid unnecessary loading. This means that the catalysts are practically amorphous to X-ray diffraction (XRD). Study of the particles by transmission electron microscopy (TEM) is handicapped by contrast from the support as is demonstrated in figure 1 . In general particles of sizes below $0.7 \mathrm{~nm}$ are impossible to detect.

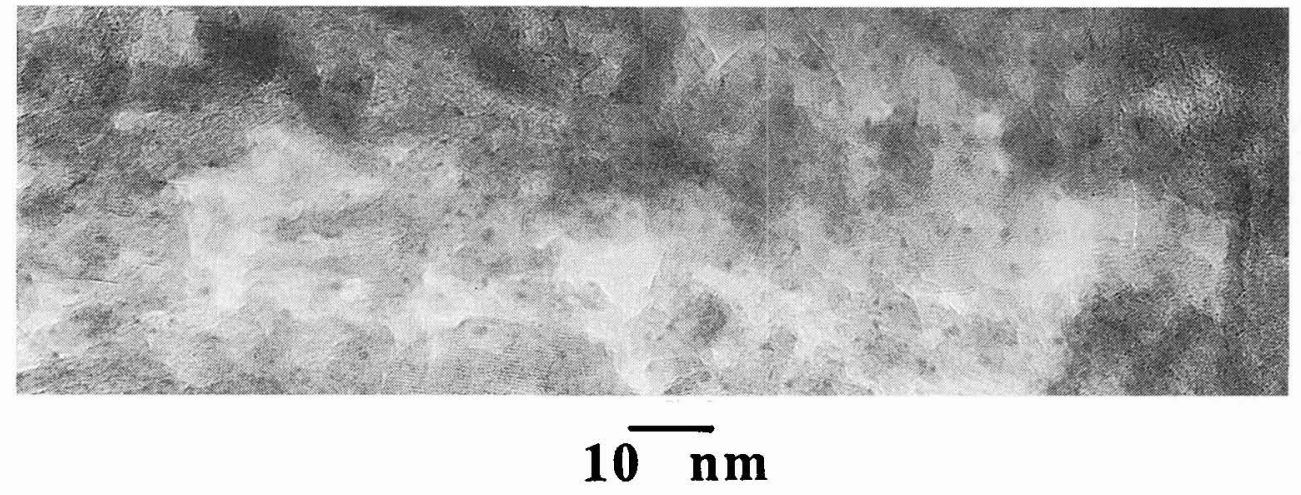

Figure 1. A typical reforming catalyst with nanometre scale particles on a poorly organised alumina support.

Typical industrial loadings are 0.3 to 1 weight per cent. X-Ray photoelectron spectroscopy (XPS ) may give useful information on the electronic state of the metals. The disadvantages of TEM and XPS requiring ultra - high vacuum are however well recognised.

The sulfide particles are in general larger, partly due to the higher loadings ( ten to twenty per cent ) possible for this type of catalyst. With TEM and XRD one is able to define the basic structure as twisted books of MoS2 sheets a few " pages " thick. The location of the promoter atoms (inserted into the sheets or as a decoration ) is not possible by these methods. 


\section{INFORMATION FROM XAS}

It is not possible here to review XAS theory, for which there are several informative works [2]. Some comments on aspects specific to industrial catalyst studies are however relevant. XAS provides both structural information ( from the extended fine structure or EXAFS ) and electronic information (from the near edge structure, XANES ) on specific elements in a sample. It is not a routine analysis tool however and cannot be used, as for instance electron microscopy is, for control of specific batches of industrial catalysts. The type of infomation we can aim at for industrial use is therefore more of a generic nature, enabling conceptual models to be built in order to understand how a class of catalysts works and how it can be improved. Although XAS can be deliberately made surface sensetive by the use of special detection systems, in the classical transmission set - up it is a bulk analysis technique. Since, however, the catalysts we are considering have been designed so that the surface is the dominant feature, even a bulk analysis will thus provide information on relevent surface properties. In addition the supported metal and sulfide catalysts may seem to have been designed for XAS analysis since the alumina support adds only a small contribution to the overall absorption. XAS does not require high vacuum, indeed the experiments may be performed under controlled atmospheres with pressures of several bars for elements with edges in the energy range over $10 \mathrm{keV}$. Several teams working on XAS studies of catalysts have constructed cells enabling the reaction of interest to be carried out during spectum acquisition, in some cases the reaction rate can be controlled by gas chromatography so that for instance deactivation may be monitored [1,3].

One major handicap to XAS analysis however is that one sees an average over the whole specimen. Close control of the range of particle sizes is necessary in order to provide interpretable data. In addition, as the temperature is increased, the thermal disorder becomes important. Recently molecular dynamics simulations, taking into account the increased freedom of movement of atoms at the surfaces of small particles, have shown that standard EXAFS analysis underestimates coordination number and therefore particle size, with the effect being more pronounced as the temperature increases [4]. It is interesting to note that this work was able to be verified by simultaneous XRD using a combined in-situ set up. The possibility of combining XAS with classic or anomalous XRD to investigate both short range and long range order opens up new fields of investigation. EXAFS at high temperatures on catalysts is often limited to the analysis of the first coordination shell. Information on the particle shape, or more generally on the three dimensional arrangement of atoms, cannot then be extracted. The type of structural model which can be built from EXAFS data is in these cases fairly qualitative, often a proposed model is only one of several which may fit the data. Structural constraints on the model imposed by XRD, as for those imposed on the chemical composition by X-ray fluorescence and on the size by TEM enable a closer approach to the reality. Thus XAS cannot hope to replace classic laboratory techniques but must work in harmony with them. The major role of XAS stems from the fact that the information provided, even that which must be interpreted semi-quantitatively, is often not available from any other technique.

\section{EXAMPLES OF APPLICATIONS TO CATALYSTS}

As for XAS theory, it is not possible to cover the whole field of applications of the technique to catalysts. Several reviews exist [5] and the major EXAFS conferences contain special sections on catalyst applications. The two examples treated here demonstrate how even a qualitative overview of XAS data can be of precious help in constructing a model of a catalyst.

\subsection{Sulfides}

As mentionned above, XAS analysis of these catalysts is facilitated by the relatively high metal loading. The active phase is achieved by sulfuration of oxides under a $\mathrm{H}_{2}+\mathrm{H}_{2} \mathrm{~S}$ mixture. The particles are highly reactive and although sealed sample holders may be loaded in a glove box, analysis is best carried out insitu after sulfuration. This means in general a dedicated apparatus since sulfur is likely to adsorb onto the walls of the cell and is a poison for other catalysts such as metals. The type of information provided by EXAFS is illustrated in figure 2 by a study at the tungsten $L_{\text {III }}$ edge of model tungsten on alumina based catalysts, one of which is promoted by nickel. Although tungsten loading and sulfuration conditions are the same for the two catalysts, simple visual inspection and comparaison with $\mathrm{WS}_{2}$ and $\mathrm{WO}_{3}$ references reveals that the unpromoted tungsten is not completely sulfided.

An estimation of the degree of sulfidation may be obtained from the ratio of W-O to W-S bonds. In the case of the promoted catalyst one also observes a lower co-ordination to sulfur for the first shell ( 5 instead of 6 for $\mathrm{WS}_{2}$ ), but especially for the higher shells due to the small particle sizes. Although particle sizes may easily be obtained by TEM for these systems, the incomplete first shell co-ordination of the major active metal is not detectable by other means and suggests an explanation for the catalytic activity in desulfuration. The activation of the catalyst may be followed by in-situ as shown by figure 3 from a study at the Mo $\mathrm{K}$ edge of a Ni-Mo/alumina catalyst under flowing $\mathrm{H}_{2}+\mathrm{H}_{2} \mathrm{~S}$ mixture. The decrease in Mo-O coordination accompagnied by the formation of Mo-S bonds can be followed as a function of temperature. 

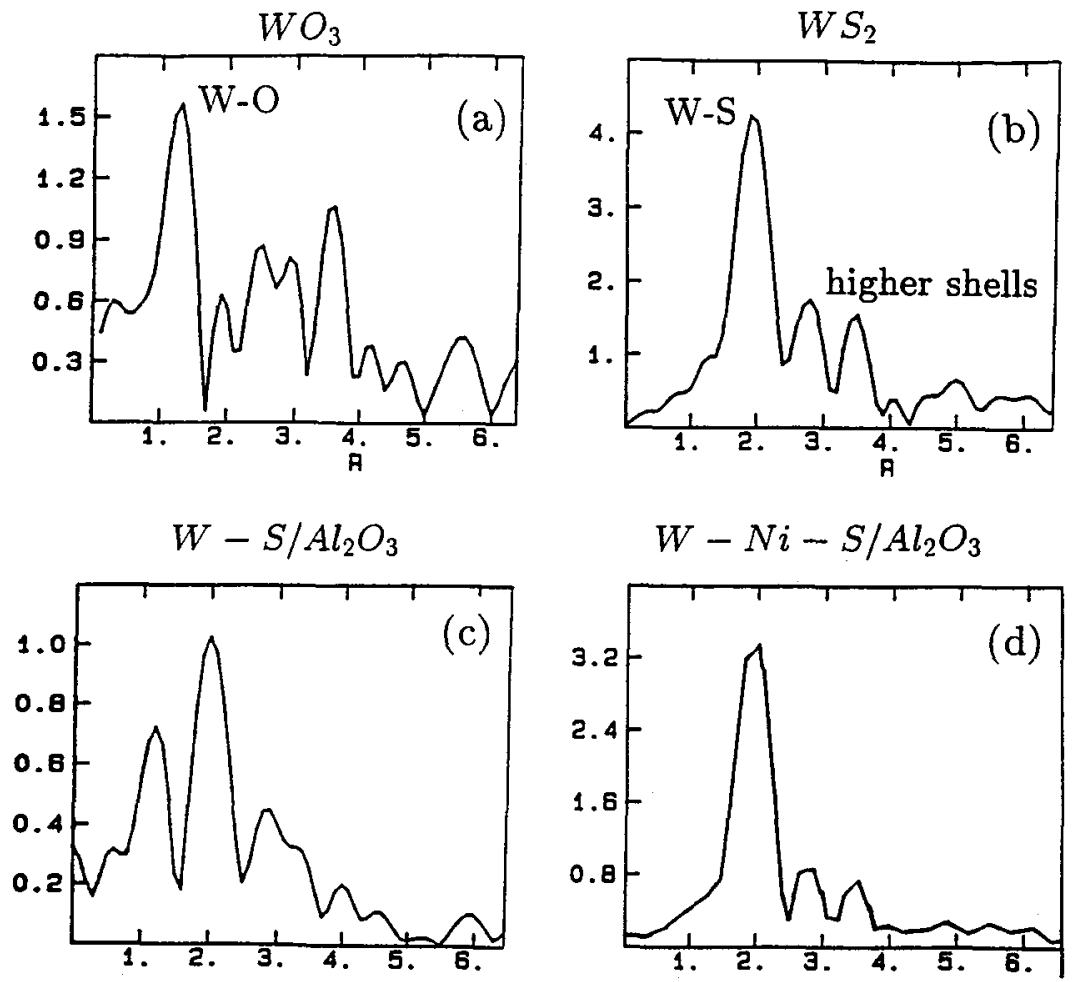

Figure 2 Comparison with reference oxide and sulfide compounds of unpromoted and Ni promoted sulfided tungsten on alumina catalysts ( $\mathrm{x}$ axis in $\AA$ ).
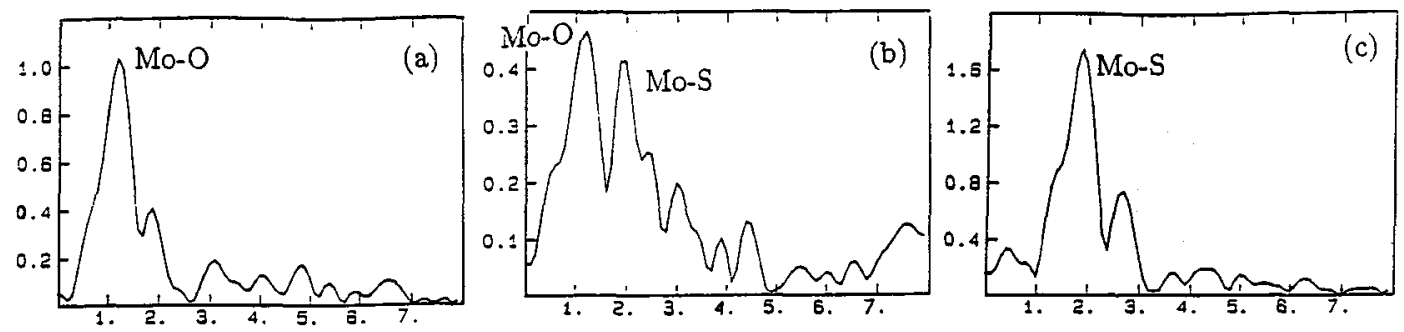

Figure 3 Evolution of a Mo-Ni/alumina catalyst during sulfidation at (a) $20^{\circ} \mathrm{C}$, (b) $200^{\circ} \mathrm{C}$, (c) $400^{\circ} \mathrm{C} \mathrm{(x}$ axis in $\AA$ ).

The promotor environnement has been studied at the Ni $\mathrm{K}$ edge for different nickel loadings expressed as $\alpha=\mathrm{Ni} /(\mathrm{Ni}+\mathrm{Mo})$ from 0.05 to 0.95 . The results shown in table 1 are coherent with the formation of nickel sulfides for high loadings ( nickel in $\mathrm{NiS}_{2}$ has a sulfur coordination of 6 at $0.237 \mathrm{~nm}$ ). This is indeed confirmed by XRD.

At lower loadings where no Ni containing structure is observer by DRX, the EXAFS results suggest the approach to an analogy to the Muiller complexes [6] based on the structural element $\mathrm{Mo}_{2} \mathrm{NiS}_{6}$ with a Ni $S$ distance of $0.219 \mathrm{~nm}$ and a coordintaion number of four. Returning to the reactivity of these solids, it is noteworthy that on contact with air the return to an oxidised, sulfate like, environnement is visible first in the Ni EXAFS, the change in the Mo coordination being slower. This strongly supports the idea of a decoration of the $\mathrm{MoS}_{2}$ sheets by surface nickel. 
Table 1 Coordination numbers $\mathrm{N}$ and distances $\mathrm{R}$ for nickel at various loadings in sulfided Ni-Mo on alumina catalysts.

\begin{tabular}{|c|c|c|}
\hline $\begin{array}{c}\alpha \\
=\mathrm{Ni} /(\mathrm{Ni}+\mathrm{Mo})\end{array}$ & $\begin{array}{c}\mathrm{N} \\
( \pm 1)\end{array}$ & $\begin{array}{c}\mathrm{R} \\
(\mathrm{nm} \pm 0.005)\end{array}$ \\
\hline 0.05 & 5 & 0.220 \\
0.2 & 4 & 0.223 \\
0.3 & 5 & 0.225 \\
0.6 & 6 & 0.235 \\
0.9 & 5 & 0.237 \\
\hline
\end{tabular}

\subsection{Supported metals}

The activation of these catalysts provides another example of the possibilities of in-situ studies. The intensity of the white line XANES feature on the LIII edge has been linked to the density of empty d type electron states [1]. By the use of reference compounds, for which the $d$ band electron density has been calculated, it is possible to calibrate the intensity in terms of empty states. It is thus possible to follow the approach to the metallic state of a calcined sample of Pt/alumina for which the EXAFS shows the local coordination of the platinum to be six oxygens.

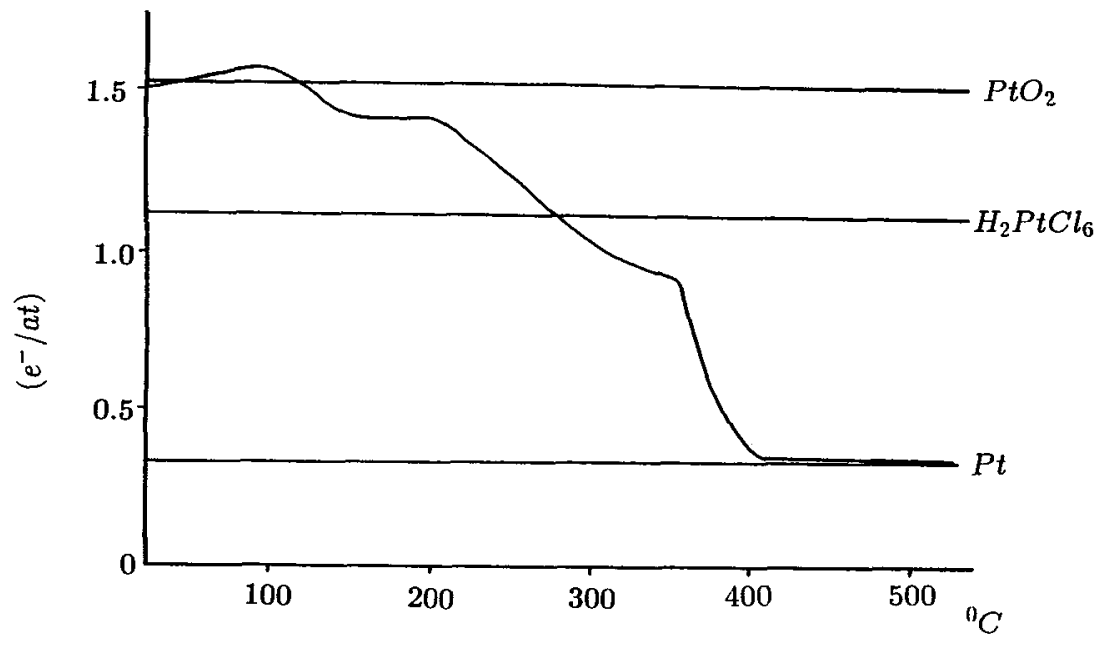

Figure 4 The number of empty $\mathrm{d}$ band states as a function of temperature for a $\mathrm{Pt} /$ alumia catalyst under flowing hydrogen. The horizontal lines represent data from bulk reference compounds.

In figure 4 one observes a slow reduction of the platinum under hydrogen as the temperature is raised to $350^{\circ} \mathrm{C}$. At this temperature the empty $\mathrm{d}$ band state density is equivalent to 0.9 electrons par atom, comparable to the theoretical value of one in the $5 \mathrm{~d}^{9}$ configuration of an isolated platinum atom. There follows a sharp fall to the value of bulk platinum $\left(0.33 \mathrm{e}^{-/} / \mathrm{atom}\right)$ by $400^{\circ} \mathrm{C}$. This simple example can be compared directly with temperature programmed reduction (TPR) of the catalyst to confirm the findings. In the case shown here one does indeed observe the major peak of hydrogen consumption in the range of $300^{\circ} \mathrm{C}$ to $400^{\circ} \mathrm{C}$. In the case of bimetallic catalysts, where it is not possible to attribute TPR peaks directly to one or other of the metals, the element specificity of XAS is highly valuable.

For the platinum phase formed by high temperature reduction, the simplest model that fits the EXAFS data is of a particle containing 10 to 20 atoms, based on the bulk fcc structure. No firm evidence for bonding to the support oxygens is provided : the metal - support interaction may be weak or may be affected by a large statistical distribution of distances. These considerations are once again only semi quantitative but help to eliminate a large number of possible models incompatible with the EXAFS results.

In the study of bimetallic catalysts a major question to be adressed is the formation or not of an alloy phase. The preparation procedures normally used, involving successive impregnation or mixtures of salts of the two metals, do not guaranty the formation of an alloy and modifications to the catalytic activity may be interpreted as due to reactions occuring first on one monometallic phase then on another, close 
by on the support but not in intimate contact. One example in which the formation of an alloy is evident from the EXAFS results is a model Pt-Zr catalyst. Here the particles are fairly large, around $10 \mathrm{~nm}$ in diameter, leading to high signal to noise ratios in the data. Figure 5 shows the pseudo - radial distribution functions around platinum of a bimetallic $\mathrm{Pt}-\mathrm{Zr}$ and a monometallic $\mathrm{Pt} /$ alumina catalyst prepared so as to have the same particle size.
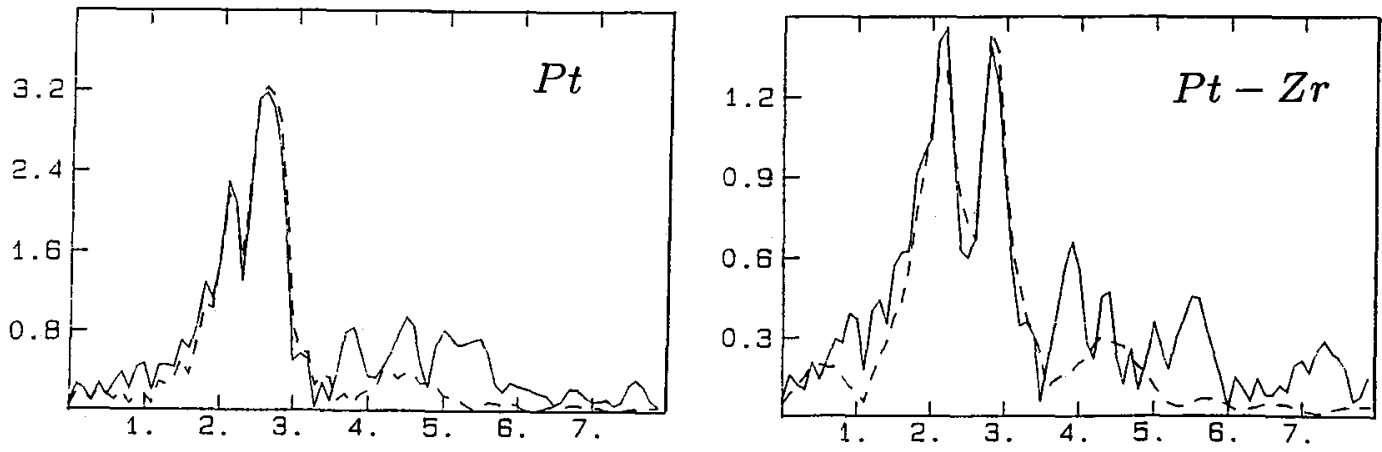

Figure 5. Pt LIII edge data for monometallic Pt and bimetallic Pt-Zr catalysts ( $x$ axis in $\AA$ ).

The large difference in the distributions comes from the phase difference between platinum and zirconium neighbors leading to a beat in the EXAFS spectrum and a splitting of the first neighbor peak. The fit to the data in the $\mathrm{Pt}-\mathrm{Zr}$ case, with four $\mathrm{Zr}$ and eight $\mathrm{Pt}$ neighbors at $0.285 \mathrm{~nm}$, is in close accord with the hypothesis of formation of a $\mathrm{Pt}_{3} \mathrm{Zr}$ alloy. Again no interaction with the support is detected by EXAFS, although for large particles this is not surprising as any metal atoms at the interface will be in a minority.

The above case, with large particles, is for EXAFS practically equivalent to the analysis of bulk samples. The acquisition times used were of the order of ten minutes. As the particle size decreases the number of neighbors decreases and for bimetallic systems they may be of two types when an alloy is formed. For realistic systems, with subnanometer particles, one is thus faced with the need to extract more information from data where the signal to noise ratio is intrinsically lower. Acquisition times are thus much longer, of the order of hours.

In contrast to the monometallic and the large particle cases for which no particle - support interface can be defined from EXAFS, for bimetallic Pt-Sn catalysts recent work [7] has shown the existance of Pt-O bonds as well as Pt-Sn and Pt-Pt bonds in the reduced solid. The XANES of platinum confirms that the element is in a reduced state. Table 2 resumes the structural parameters deduced for monometallic and bimetallic catalysts after calcination ( the results are identical for the two catalysts ) and after reduction. The EXAFS results provide not only proof of intimate contact between the two metals but also evidence for an improved particle - support contact, which is sufficiently well defined to be detected.

Table 2. Platinum $L_{\text {III }}$ EXAFS results for calcined and reduced supported metal catalysts (estimated precision is $15 \%$ for the coordination number $\mathrm{N}, 0.002 \mathrm{~nm}$ for the distance $\mathrm{R}$ ).

\begin{tabular}{|c|c|c|c|c|c|c|}
\hline \multirow{2}{*}{$\begin{array}{c}\text { nearest } \\
\text { neighbor }\end{array}$} & \multicolumn{2}{|c|}{$\begin{array}{c}\text { calcined } \\
\text { Pt or Pt-Sn }\end{array}$} & \multicolumn{2}{|c|}{$\begin{array}{l}\text { reduced Pt/alumina } \\
\text { monometallic }\end{array}$} & \multicolumn{2}{|c|}{$\begin{array}{l}\text { reduced Pt-Sn / alumina } \\
\text { bimetallic }\end{array}$} \\
\hline & $\bar{N}$ & $\mathrm{R}(\mathrm{nm})$ & $\mathbf{N}$ & $\mathrm{R}(\mathrm{nm})$ & $\bar{N}$ & $\mathrm{R}(\mathrm{nm})$ \\
\hline 0 & 6 & 0.201 & 0.0 & $=$ & 1.9 & 2.24 \\
\hline $\mathrm{Pt}$ & 0 & - & 6.4 & 0.275 & 1.6 & 0.268 \\
\hline Sn & 0 & - & - & - & 1.2 & 0.293 \\
\hline
\end{tabular}

The clear increase in strength, or at least in coherence, of the metal support interaction, attributed to the presence of tin in the coordination sphere, is of considerable importance in understanding the resistance to sintering of platinum - tin catalysts, which has itself been the subjet of XAS and TEM investigation. Under repeated cycles of reduction and oxydation the monometallic catalysts loose their activity due to the formation of large particles, clearly visible in TEM and reaching sizes of several tens of nanometers. The bimetallic catalysts on the other hand retain both their activity and the high dispersion of the metal up to six cycles.

The use of in-situ cells has allowed the study of catalysts in contact with hydrocarbons at high temperatures ( $300^{\circ} \mathrm{C}$ and $460^{\circ} \mathrm{C}$ ). In a recent study [8] the behaviour of monometallic $\mathrm{Pt} /$ alumina and 
bimetallic Pt-Re/alumina samples was compared. The reforming of $\mathrm{n}$-heptane was chosen as a test reaction. When the monometallic catalyst is working ( as monitored by gas chromatography) the platinum LIII EXAFS is modified with the appearance of new bonding due to low atomic number neighbors at short distances. Analysis showed these to be carbon atoms. Although it is notoriously difficult to distinguish atoms of close atomic number, the possibility of Pt-O bonds was eliminated based on the phase behaviour of the EXAFS oscillations but also on chemical reasoning : the formation of Pt-C bonds is to be expected in a working catalyst. No other modifications to the metal particle structure were observed. In the case of monometallic catalysts $\mathrm{Pt}-\mathrm{C}$ bonds were detected ( with a coordination number of 1.2 at $0.194 \mathrm{~nm}$ ) at both temperatures studied. For the bimetallic catalyst only at the lower temperature were Pt-C bonds observed.

The fact that the $\mathrm{Pt}$ - Re catalyst was still functionning at high temperature may mean that contact of the hydrocarbons is occuring only with the rhenium atoms, but the platinum - carbon interaction itself may also be strongly modified. Indeed at lower temperatures, where carbon bonds are seen, both the coordination number ( 2.0$)$ and more significantly the distance $(0.209 \mathrm{~nm})$ are different. A modification of such magnitude in the Pt-C bond length seems to indicate a change in the type of bonding in the bimetallic system compared to the monometallic case. There is indeed a significant improvement for $\mathrm{Pt}-\mathrm{Re}$ catalysts in resistance to the formation of coke, one of the major causes of deactivation in industrial applications.

\section{PERSPECTIVES FOR XAS}

The examples shown above demonstrate that significant information can be obtained from EXAFS at a qualitative level on model systems close to industrial catalysts. This information is extremely valuable in defining a general view of the structure of catalysts and how they work. The possibility to use EXAFS in-situ has enabled modifications to the structure with temperature and in contact with reactants to be followed. No attempt has been made here to develop atomic scale models in three dimensions of the catalyst particles or of their interactions with promotors and hydrocarbon molecules as this is far from being a straightforward process. Indeed, for the greater part of the systems studied, it is impossible using EXAFS alone to build such a definitive model. Two technological developpements are likely to improve the situation : acces to new high intensity sources and coupling of EXAFS to other techniques. In particular for the supported metal catalysts studied, where the loading is $1 \%$ or lower by weight, the signal to noise ratio of the data prohibits extraction of information from outer shells of coordination which would be useful in construction of a three dimensional model. The coupling of EXAFS to diffraction has already been mentioned as being of great importance in studying particle sizes. In other examples shown here, such as the activation of molybdenum sulfide catalysts, simultaneousdiffraction data would also be invaluable in identifying three dimensional phase formation.

The data presented here were acquired at the LURE facility in Orsay in collaboration with $\mathrm{H}$. Dexpert and D. Bazin to whom many thanks are due.

\section{References}

[1] Lytle F.W. et al. J. Chem. Phys. 70 (1979) 4849-4855

[2] Stern E.A. and Heald S.M., Handbook on Synchrotron Radiation, Vol. 1 (North Holland Publishing Co., Amsterdam, 1983) pp. 955-1014

[3] Guyot - Sionnest N.S. et al. Catal. Lett. 8 (1991) 283-296 and 297-304

[4] Clausen B.S. et al. J. Catal. 141 (1993) 368

[5] see for instance Bart J.C.J. and Vlaic G. Adv. in Catalysis 35 ( 1987) 1-138

[6] Muller A. et al. Angew. Chem. Int. 20 (1981) 934-938

[7] Caballero A. et al. J. Phys. Chem. 97 (1993) 11283-11285

[8] Caballero A. et al. J. Chem. Soc. Farad. Trans. 89 (1993) 159-164 